\title{
Clinical Trial Site Management Meeting Material
}

National Cancer Institute

\section{Source}

National Cancer Institute. Clinical Trial Site Management Meeting Material. NCI

Thesaurus. Code C115603.

A collection of agendas, presentation materials, and other documents generated for attendees during a clinical trial site management meeting. 